\title{
Simulation of linear gradient lenses for subwavelength focusing of Gaussian beams
}

\author{
Savelyev D.A. \\ Samara State Aerospace University, \\ Image Processing Systems Institute, Russian Academy of Sciences
}

\begin{abstract}
This paper demonstrates the use of linear gradient lenses (a diverging lens and a converging lens) for subwavelength focusing of Gaussian beams. Numerical simulations have shown that increasing the length lens provides a more compact focal region in both the transverse and longitudinal directions.
\end{abstract}

Keywords: subwavelength focusing, linear gradient lense, FDTD, Gaussian beams

Citation: Savelyev D.A. Simulation of linear gradient lenses for subwavelength focusing of Gaussian beams. Proceedings of Information Technology and Nanotechnology (ITNT-2015), CEUR Workshop Proceedings, 2015; 1490: $97-$ 104. DOI: $10.18287 / 1613-0073-2015-1490-97-104$

\section{Introduction}

The subject of the study gradient optics is phenomena associated with optical effects in media with a gradual change of the refractive index [1]. Medium consisting of materials which are of the type GRIN (GRadient INdex), have distributed on the gradient refractive index material [2]. Thus, light rays are bent in a curve passing through them, which allows using them for the best focus [3-6]. In [3] used hyperbolic planar lens, which allowed to focus the light in the focal spot with a diameter of the half-width at half intensity (FWHM) equal 0,131 $\lambda$, in [4] dealt with a 2-D gradient microlenses Mikaelian, in [5-6] - two-dimensional photonic crystals. Photonic crystals due to their properties are used for several applications [7], among which their application to optical waveguides [8-9].

Currently in the integrated and fiber optics there is a large variety of optical waveguides with different properties. Planar waveguides and fibers along the profile of the spatial distribution of the refractive index can be divided into two groups: with step refractive index profile and with gradient profile [10-11]. In the case of a gradient profile refractive index varies smoothly from the center of the waveguide to the boundary defined by the law [11].

One of the major advantages of the lenses belonging to the gradient optics is that the optical surfaces of gradient lenses may be flat, which is important for the inputoutput of radiation of the fiber.

It is known that high-order mode exhibit greater divergence, however, the use of multimode method is one of the possible ways to increase the capacity of modern 
communication systems [12]. And the most promising is the multiplexing of modes with different orders of the vortex phase singularity [13-14].

Distribution and generation of free-space laser vortices studied quite well [15-18], in [19-21] shows how to use them to focus the simple micro-elements of square and cylindrical shape. However, obtaining an optical fiber separate vortex modes and their superpositions is a big problem [14, 22-26]. In contrast to the classic LP-mode (mode optical fibers), angular harmonics are invariant to the scale at the input and output of fiber with the help of diffraction microstructures. This gives greater freedom in choosing the parameters of the optical circuit. In [27] have been identified application features sharp focusing of laser modes for introduction into a fiber of smaller diameter with using the binary micro-relief applied at the output end of the optical fiber. Overlaying certain conditions can generate laser light having a property of reproducible [28], while the phase shift between the modes at certain distances to approximate the desired cross-sectional intensity distribution of the laser beam [29].

In this paper, numerically investigate the diffraction of Gaussian beams on a linear gradient lens with different length. For the numerical simulation of diffraction of the considered laser beams using finite-difference time-domain method (FDTD), implemented in the software package Meep [30]. Numerical simulations were performed using the computing power of a cluster 775 GFlops. Features a cluster: the number of cores -116 , computes nodes: 7 twin servers HP ProLiant 2xBL220c, the amount of RAM 112 GB.

a)
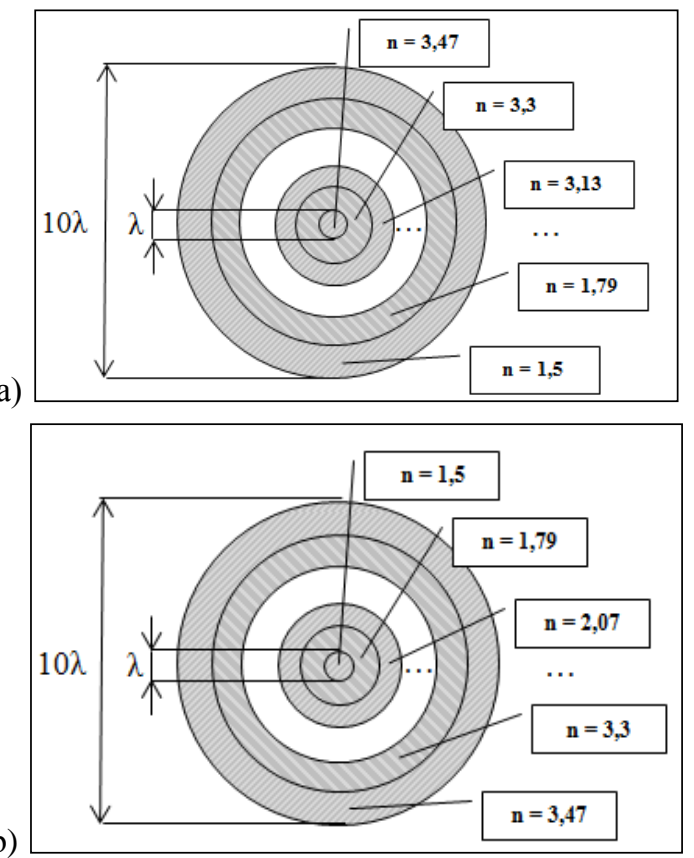

Fig. 1. - The transverse structure of the considered optical element: a) converging lens, b) diverging lens 


\section{Investigation of diffraction of Gaussian beams}

In the paper considers two types of laser beams, which retain their structure during propagation in the free space in the circular polarization: a fundamental Gaussian mode, mode Gauss-Laguerre $(0,1)$.

Figure 1 shows the transverse structure of the considered optical trace element (diverging lens and the converging lens). Later in the linear gradient lenses, in which the refractive index decreases from the central part to the edges of the lens will be called converging lens. The refractive index gradient lenses varies linearly as: all considered 10 rings (width $\lambda$ ), each of which had a their refractive index (from $n=$ 3.47 on the center, until $n=1.5$ on the edge of the lens).

a)

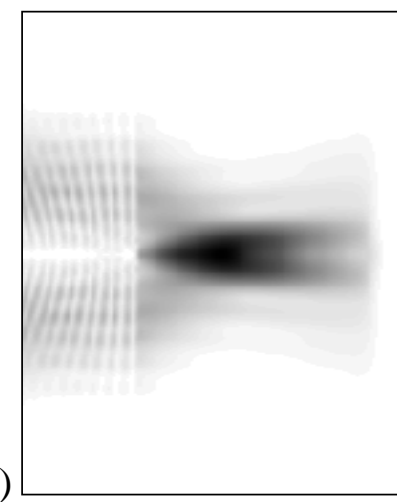

b)
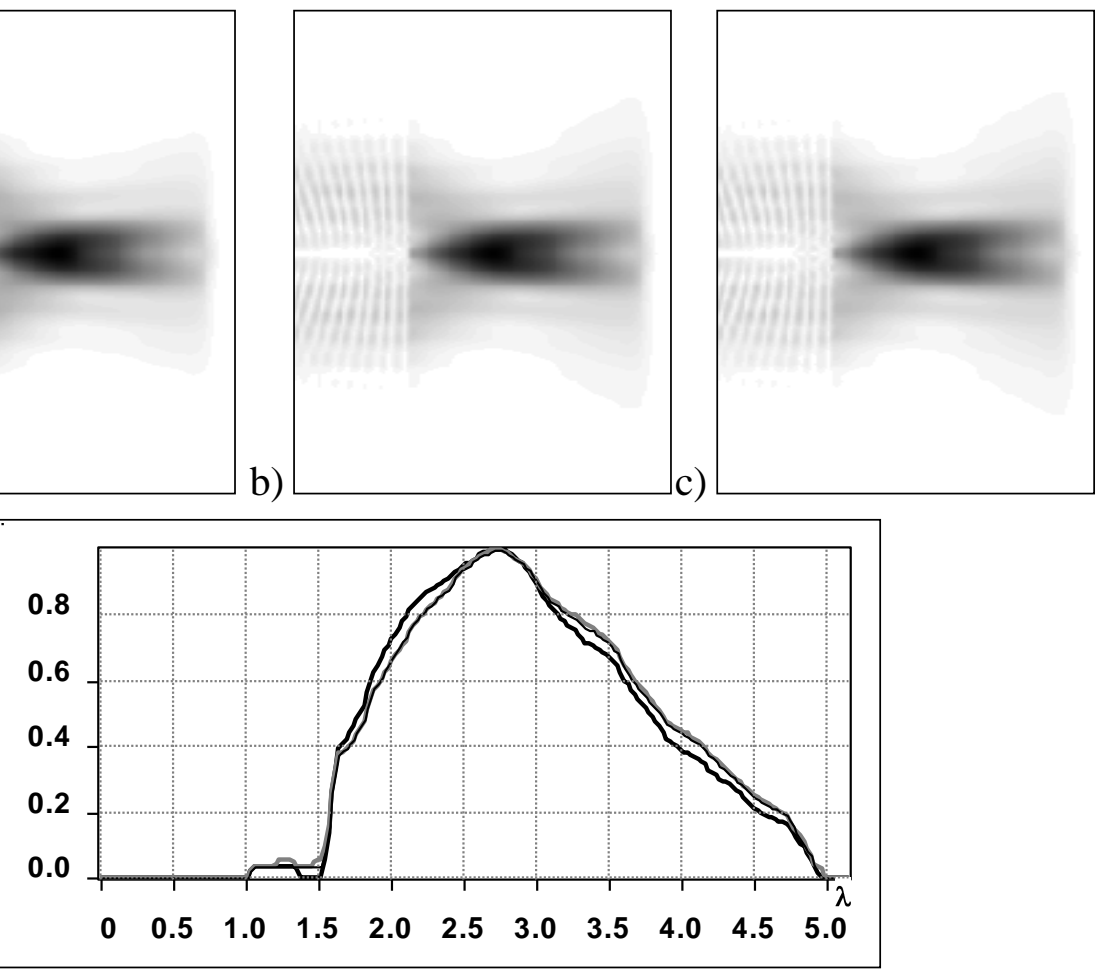

d)

Fig. 2. - Diffraction mode Gauss - Laguerre $(0,1)$ on the converging linear gradient lens, the overall intensity of the: a) 40 tacts, b) 60 tacts, c) 80 tacts, d) the schedule of general axial intensity, thick black lines - 40 tacts black fine line -60 tacts, gray line - 80 tacts

Simulation parameters: the wavelength $\lambda=1.55$ microns, the size of the computational domain $x, y, z \in[-4.5 \lambda ; 4.5 \lambda]$. The thickness of the absorbing layer PML $\sim 0.65 \lambda$ ( 1 micron), the sampling step of space $-\lambda / 31$, the sampling step of time $-\lambda /(62 c)$, where $c$ is the velocity of light. Simulation time - 40 tacts (one for that meant the propagation time over a single wavelength), and was chosen so that the kind of laser radiation did not change with increasing simulation time (Figure 2).

The size of the focal spot on the FWHM considered a global maximum (maximum intensity). Table 1 shows the diffraction of a fundamental Gaussian mode and mode 
Gauss-Laguerre $(0,1)$ on the converging lens when you change the length $L$. Considered the overall intensity.

The table shows both the diffraction pattern and the size of the focal spot, that with increasing length of the focusing lens has been increasing. If $L=0.25 \lambda$ presence lens has almost no effect, then $L=2 \lambda$ diffraction pattern has changed significantly: the size of the focal spot on the half-width at half intensity decreased by 2.67 times (from FWHMmax $=1.95 \lambda$ for $L=0.25 \lambda$, to FWHMmax $=0.73 \lambda$ for $L=2 \lambda$ ). Comparing 4 and 5 columns of Table 1, it can be seen that the depth of focus, decreased significantly with a slight reduction of the transverse dimension of the focal spot in both these cases. Increasing the length of the lens results in better focus, it is clearly seen when comparing the first and last column of Table 1 for the case of fashion Gauss - Laguerre (0.1). Note that FWHMmax also decreases with increasing length of the lens. Also the global maximum (max) also considered at the edge section of the optical element (out).

Table 1. Diffraction of Gaussian beams on the converging linear gradient lens, the overall intensity

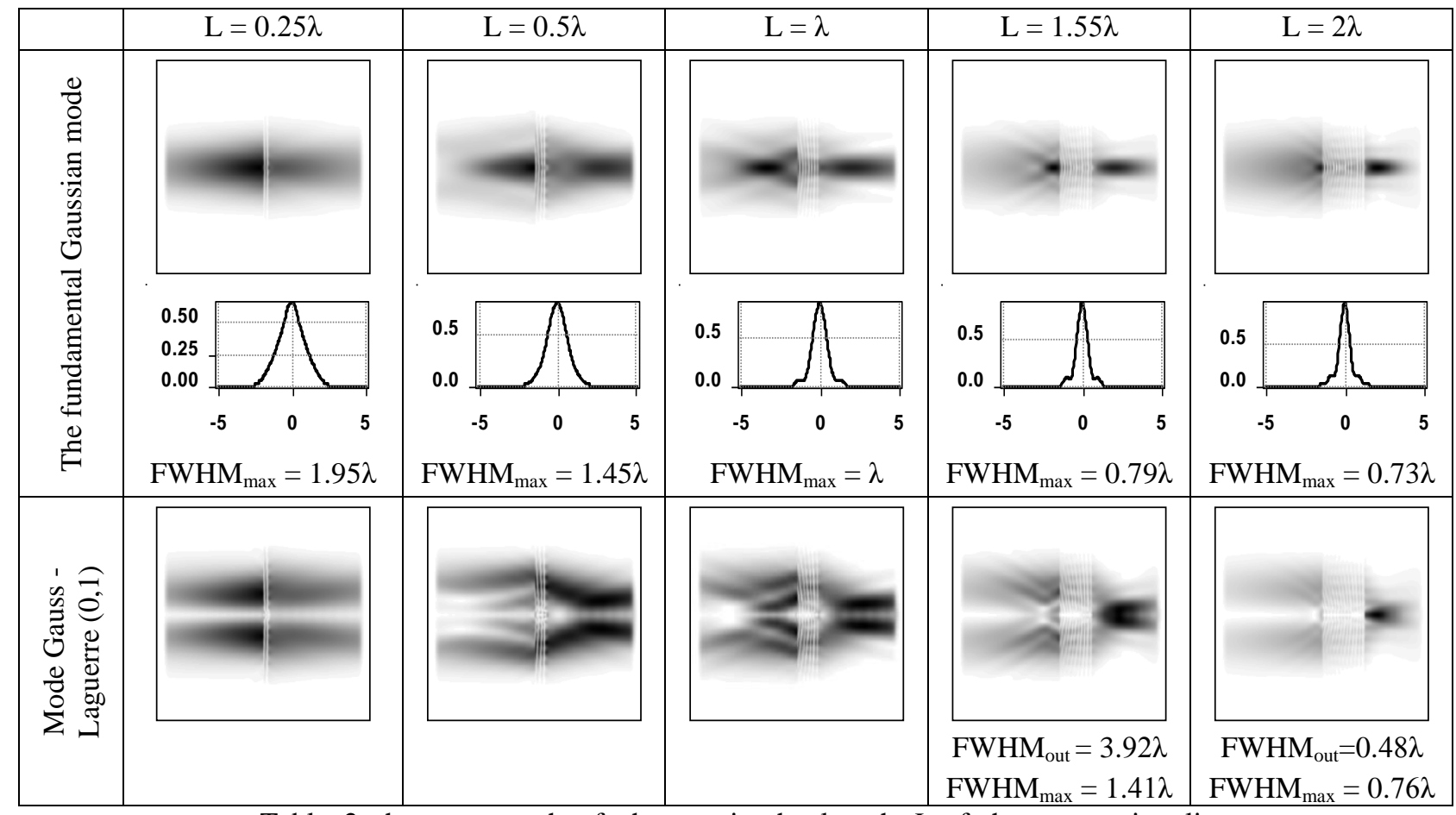

Table 2 shows research of changes in the length $L$ of the converging linear gradient lenses separately dedicated to the longitudinal component of the electric field. In addition to the global maximum ( $\max$ ) is considered the first maximum of the optical element (fmx). 
Table 2. Diffraction of Gaussian beams on the converging linear gradient lens, the intensity of longitudinal component

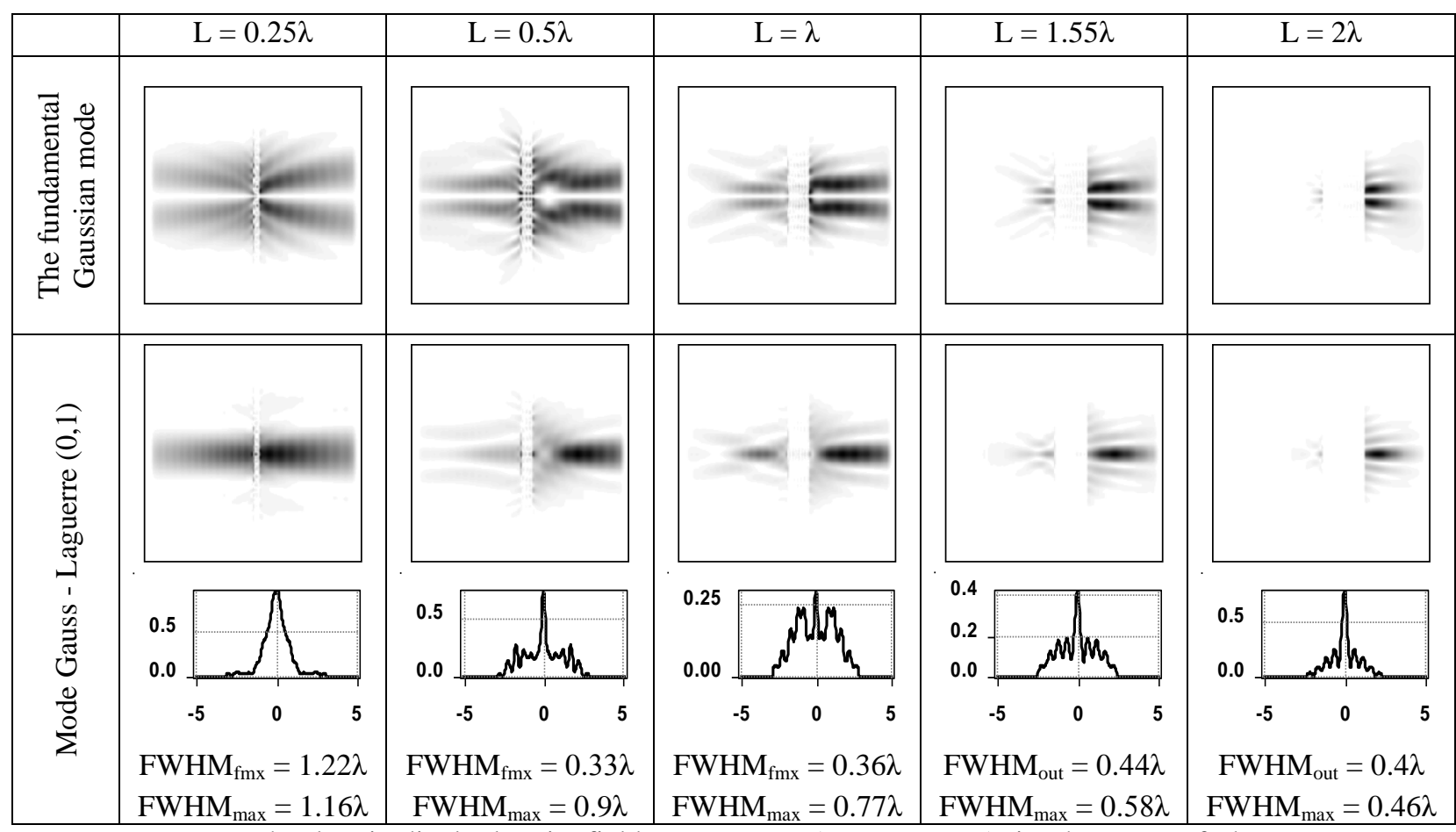

The longitudinal electric field component (component $z$ ) in the case of the fundamental Gaussian mode also becomes smaller with increasing length of the lens. Comparing Tables 1 and 2 for the case of mode Laguerre - $(0,1)$, it should be noted that increasing the length of the lens, there is a significant enhancement $z$ components, and the size of the focal spot begins to form mainly due to the longitudinal component of the electric field. This is particularly noticeable when analyzing the last column of table 1 and 2: for mode Gauss - Laguerre $(0,1)$ size of the focal spot next to the central part of which contains the longitudinal component of the electric field is $\mathrm{FWHM}_{\text {out }}=$ $0.4 \lambda$, while the total intensity of the light Spot contains a cross-polarized side lobes that broadens the spot size to FWHM $=0.48 \lambda$. In the plane of maximum intensity, the effect of cross-polarized components of the electric field is more significant (broadened to $0.76 \lambda$ ). Of special note is the case of $L=0.5 \lambda$, when the longitudinal component of the electric field near the plane of the first element in the maximum was obtained by the minimum size of the focal spot: $\mathrm{FWHM}_{\mathrm{fm}}=0.33 \lambda$, but as you can see on the the graphs - by increasing the intensity of the side lobes.

Table 3 shows the result of diffraction of Gaussian beams at the diverging linear gradient lens when changing the length $L$, that is, on the lens, whose refractive index increases from the center to the edges. Such a lens can be used for the introduction of laser radiation into a hollow (annular) optical waveguide. The refractive index in this case, $i$ have a similar case considered earlier: also examined 10 rings (width $\lambda$ ), each 
of which had a their refractive index. But they are changed in the reverse order of $n=$ 1.5 in the middle, to $n=3.47$ at the edge of the lens (Figure 1b).

Table 3 shows that the longitudinal component of the electric field using GaussLaguerre modes $(0.1)$ in the plane of maximum intensity able to obtain a focal spot size smaller than the collecting lens, and in the case of $L=1.55 \lambda$ size of the focal spot is the focal spot in the case of $L=2 \lambda$ in Table 2 . However, the intensity of the spots materially $\sim 5$ times less.

Table 3. Diffraction of Gaussian beams on the diverging linear gradient lens

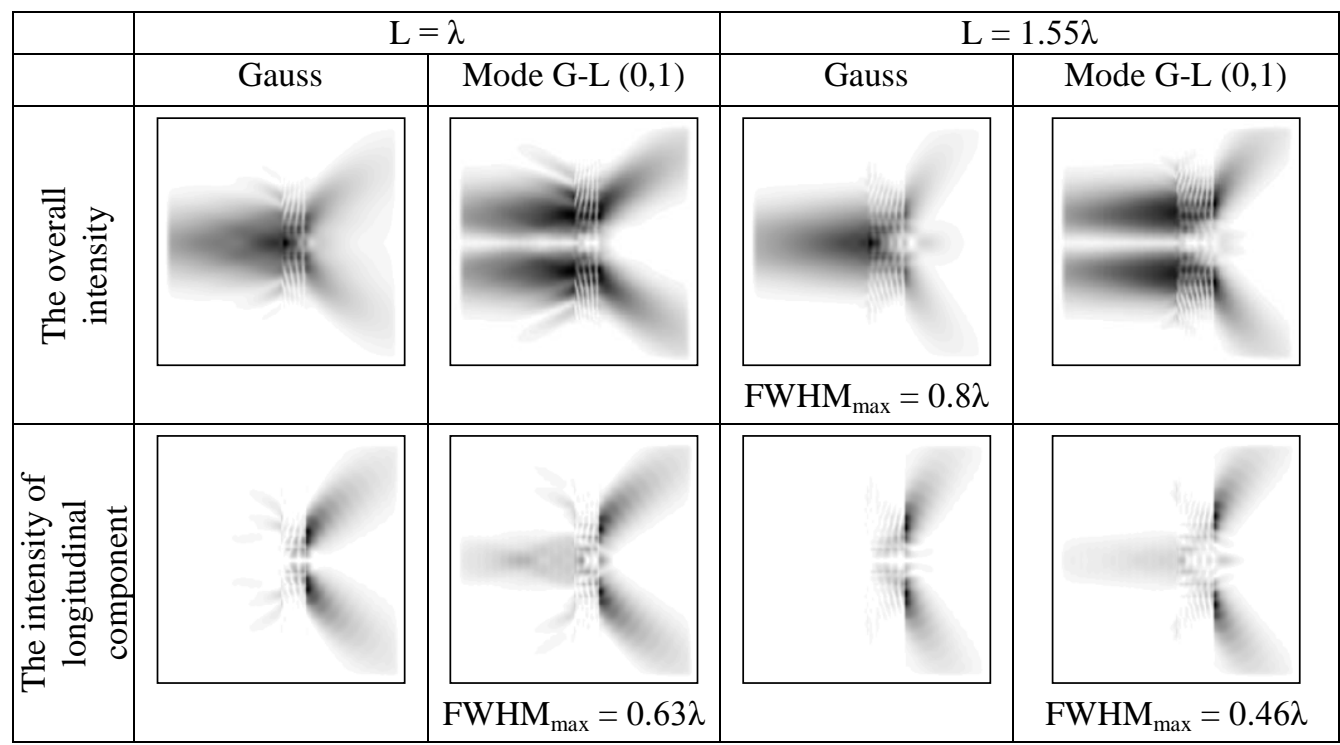

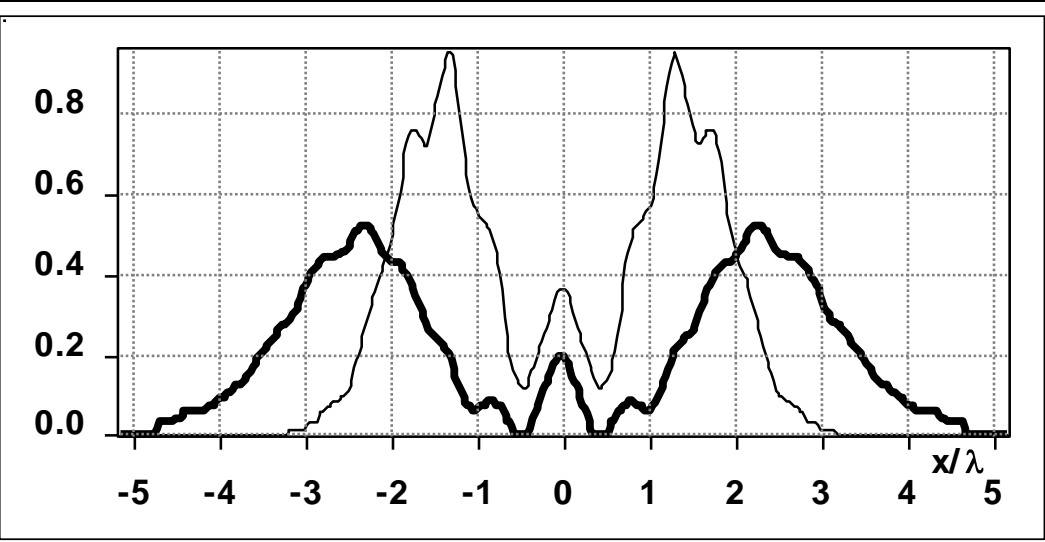

Fig. 3. - Graphics cross-section of the intensity of the longitudinal component for modes Gauss - Laguerre $(0,1)$ on the diverging linear gradient lens, black thick line $-h=1.55 \lambda$, black thin line $-h=\lambda$ 
Figure 3 is a graph showing the cross section of the components of $z$ to LaguerreGaussian modes $(0,1)$ (bottom row of Table 3). As seen from the graph, a reduction intensity of the focal spot accompanied by decrease intensity of side lobes.

\section{Conclusion}

In this paper using the FDTD method, was numerically research of circularly polarized Gaussian beams passing through a linear gradient lens, considered two options of lenses - converging and diverging.

It has been shown that increasing the length lens provides a more compact size of the focal region in all directions, both for total intensity and the longitudinal electric field component.

The minimum size of the focal spot was not obtained at the maximum intensity, but at the first maximum out of optical element. For modes Gauss-Laguerre $(0,1)$ the minimum size of the focal spot on the FWHM for the longitudinal electric field component was 0.33 wavelength for $L=0.5 \lambda$; in the plane of maximum intensity using a diverging lens for $\mathrm{z}$ components able to obtain a focal spot size less than when using a converging lens (at falling intensity $\sim 5$ times).

\section{Acknowledgements}

The work was partially funded by the Russian Foundation of Basic Research Grant (№ 14-07-31079 mol_a) and Russian Federation Ministry of Education and Science.

\section{References}

1. Tomlinson WJ. Applications of GRIN-rod lenses in optical fiber communication systems. Applied Optics, 1980; 19(7): 1127-1138.

2. Emkey W, Jack C. Analysis and evaluation of graded-index fiber lenses. Journal of Lightwave Technology, 1987; 5(9): 1156-1164.

3. Kotlyar VV, Kovalev AA, Triandafilov YaR, Nalimov AG. Modes of planar gradientindex hyperbolic secant waveguide. Computer optics, 2010; 34(2): 146-154.

4. Kotlyar VV, Kovalev AA, Nalimov AG. Gradient-index element of micro-optics for super-resolution. Computer Optics, 2009; 33(4): 369-378.

5. Matsumoto T. Focusing of light by negative refraction in a photonic crystal slab superlens on silicon-on-insulator substrate. Optics letters, 2006; 31(18): 2786-2788.

6. Kurt H. The focusing effect of graded index photonic crystals. Applied Physics Letters, 2008; 93(17): 171108.

7. Zhu Q, Jin L, Fu Y. Graded index photonic crystals: A review. Annalen der Physik, 2015; 527(3-4): 205-218.

8. Xiong C. Slow-light enhanced correlated photon pair generation in a silicon photonic crystal waveguide. Optics letters, 2011; 36(17): 3413-3415.

9. Liang J. Wideband ultraflat slow light with large group index in a W1 photonic crystal waveguide. Journal of Applied Physics, 2011; 110(6): 063103.

10. Goncharenko A, Karpenko V, Goncharenko I. Fundamentals of the theory of optical waveguides. Minsk: Belarusian science; 2009.

11. Snyder AW, Love J. Optical Waveguide Theory. Springer Science \& Business Media, 1983. 
12. Bozinovic N. Terabit-scale orbital angular momentum mode division multiplexing in fibers. Science, 2013; 340(6140): 1545-1548.

13. Essiambre R. Capacity limits of optical fiber networks. Journal of Lightwave Technology, 2010; 28(4): 662-701.

14. Khonina SN, Kazanskiy NL, Soifer VA. Optical vortices in a fiber: Mode division multiplexing and multimode self-imaging. Editer Dr Moh. Yasin. Recent Progress in Optical Fiber Research. InTech, 2012.

15. Savelyev DA, Khonina SN. Maximizing the longitudinal electric component at diffraction on a binary axicon linearly polarized radiation. Computer Optics, 2012; 36(4): 511-517.

16. Khonina SN, Savelyev DA. High-aperture binary axicons for the formation of the longitudinal electric field component on the optical axis for linear and circular polarizations of the illuminating beam. Journal of Experimental and Theoretical Physics, 2013; 117(4): 623-630.

17. Khonina SN, Karpeev SV, Alferov SV, Savelyev DA, Laukkanen J, Turunen J. Experimental demonstration of the generation of the longitudinal E-field component on the optical axis with high-numerical-aperture binary axicons illuminated by linearly and circularly polarized beams. Journal of Optics, 2013; 15: 085704.

18. Khonina SN, Savelyev DA, Kazanskiy NL. Vortex phase elements as detectors of polarization state. Optics Express, 2015; 23(14): 17845-17859.

19. Khonina SN., Savelyev DA., Ustinov AV. Diffraction of laser beam on a two-zone cylindrical microelement. Computer Optics, 2013; 37(2): 160-169.

20. Kotlyar VV, Stafeev SS, Feldman AYu. Photonic nanojets formed by square microsteps. Computer Optics, 2014; 38(1): 72-80.

21. Savelyev DA, Khonina SN. Numerical analysis of subwavelength focusing using a silicon cylinder. Computer Optics, 2014; 38(4): 638-642.

22. Berdague S, Facq P. Mode division multiplexing in optical fibers. Applied Optics, 1982; 21: 1950-1955.

23. Mikaelian AL. Optical Methods in Informatics. Moscow: Nauka (Science) Publishers, 1990.

24. Soifer VA, Golub MA. Laser beam mode selection by computer-generated holograms. Boca Raton, CRC Press, 1994

25. Karpeev SV, Khonina SN. Experimental excitation and detection of angular harmonics in a step-index optical fiber. Optical Memory \& Neural Networks (Information Optics), 2007; 16(4): 295-300.

26. Khonina SN, Striletz AS, Kovalev AA, Kotlyar VV. Propagation of laser vortex beams in a parabolic optical fiber. Proceedings SPIE, 2010; 7523: 75230B-1-12.

27. Savelyev DA, Khonina SN. Sharp focusing by means of binary relief at the end of the optical fiber. Proceedings SPIE, 2013; 9156: 915609-1-6.

28. Khonina SN, Kotlyar VV, Soifer VA, Paakkonen P, Turunen J. Measuring the light field orbital angular momentum using DOE. Optical Memory and Neural Networks (Allerton Press), 2001; 10(4): 241-255.

29. Almazov AA, Khonina SN. Periodic self-reproduction of multi-mode laser beams in graded-index optical fibers. Optical Memory and Neural Networks (Information Optics), 2004; 13(1): 63-70.

30. Oskooi AF. Meep: A flexible free-software package for electromagnetic simulations by the FDTD method. Computer Physics Communications, 2010; 181: 687-702. 